\title{
Development of an Electrochemical Biosensor for Sulforaphane Determination in Carmine Radish
}

\author{
Xianrong Zhou", Bo Jiang ${ }^{\#}$, Yan Zhang, Jin Shang ${ }^{*}$,Hui Yang ${ }^{*}$ \\ College of Life Science and Technology, Yangtze Normal University, Chongqing, 408100, P.R. China \\ ${ }^{\#}$ These authors contribute equally \\ *E-mail: shangjin@yznu.cn; yanghui@yznu.cn
}

doi: $10.20964 / 2017.03 .19$

Received: 7 December 2016 / Accepted: 30 December 2016 / Published: 12 February 2017

\begin{abstract}
A multifunctional composite of graphene (Gr), polydopamine (Pdop) and Ag nanoparticles (Ag NPs) was synthesized through a facile and gentle method, where Pdop was immobilized on Gr easily at room temperature and $\mathrm{Au}$ NPs was subsequently deposited through gently stirring. The electrochemical responses were investigated at the electrode modified with the composite of Ag, Pdop and Gr by sulforaphane acting as the model molecule. The results indicated that the electrode modified with Ag-Pdop-Gr composite exhibited remarkably favourable for the electron transfer kinetics compared to the glassy carbon electrodes modified with Gr or Ag NPs. At last, the proposed was applied into the simultaneous determination of sulforaphane of trace level in the specimens of carmine radish.
\end{abstract}

Keywords: Ag nanoparticles; Polydopamine; Graphene; Sulforaphane; Carmine radish

\section{$\underline{\text { FULL TEXT }}$}

(C) 2017 The Authors. Published by ESG (www.electrochemsci.org). This article is an open access article distributed under the terms and conditions of the Creative Commons Attribution license (http://creativecommons.org/licenses/by/4.0/). 